\title{
Analysis of MEG recordings from Alzheimer's disease patients with sample and multiscale entropies
}

\author{
Carlos Gómez*, Student Member, IEEE, Roberto Hornero, Member, IEEE, Daniel Abásolo, Member, \\ IEEE, Alberto Fernández, and Javier Escudero, Student Member, IEEE
}

\begin{abstract}
Alzheimer's disease (AD) is one of the most prominent neurodegenerative disorders. The aim of this study is to analyze the magnetoencephalogram (MEG) background activity in AD patients using sample entropy (SampEn) and multiscale entropy (MSE). The former quantifies the signal regularity, while the latter is a complexity measure. These concepts, irregularity and complexity, are linked although the relationship is not straightforward. Five minutes of recording were acquired with a 148-channel whole-head magnetometer in 20 patients with probable AD and 21 control subjects. Our results show that MEG recordings are less complex and more regular in AD patients than in control subjects. Significant differences between both groups were found in some MEG channels with both methods $(p<0.01$, Student's $t$-test with Bonferroni's correction). Using receiver operating characteristic curves, accuracies of $75.6 \%$ with SampEn and of $87.8 \%$ with $M S E$ were reached. Our findings show the usefulness of these entropy measures to increase our insight into AD.
\end{abstract}

\section{INTRODUCTION}

$\mathrm{M}$ AGNETOENCEPHALOGRAPHY (MEG) is a non-invasive technique that allows recording the magnetic fields produced by brain activity. It provides an excellent temporal resolution, orders of magnitude better than other methods for measuring cerebral activity, as magnetic resonance imaging, single-photon-emission computed tomography or positronemission tomography [1]. A good spatial resolution can also be achieved due to the large number of sensors. Moreover, the activity in different parts of the brain can be monitored simultaneously with whole-head equipments, such as the magnetometer used in the present study [1]. On the other hand, the magnetic signals generated by the human brain are extremely weak. Thus, SQUID (Superconducting QUantum Interference Device) sensors are necessary to detect them. In addition, magnetoencephalograms (MEGs) must be recorded in a magnetically shielded room.

Alzheimer's disease (AD) is the main cause of dementia in western countries [2] and affects approximately $11 \%$ of

Manuscript received March 28, 2007. This work was supported in part by Consejería de Educación de la Junta de Castilla y León under projects VA108A06 and VA102A06. Asterisk indicates corresponding author.

C. Gómez, R. Hornero, D. Abásolo, and J. Escudero are with the Biomedical Engineering Group at Department of Signal Theory and Communications, E.T.S. Ingenieros de Telecomunicación, University of Valladolid, Campus Miguel Delibes, 47011 - Valladolid, Spain (e-mail: cargom@tel.uva.es).

A. Fernández is with the Centro de Magnetoencefalografía Dr. PérezModrego, University Complutense of Madrid, Spain. population over 65 years [3]. A definite diagnosis of $\mathrm{AD}$ is only possible by necropsy, but a differential diagnosis with other types of dementia and with major depression is used. It includes physical and neurological examination, methods of medical imaging and mental status tests. During the last years, brain recordings analyses have been utilized as a useful tool to complete the diagnosis. Traditionally, AD patients' brain recordings were analyzed with linear techniques. Spectral analysis seems to discriminate $\mathrm{AD}$ patients from control subjects through an increased EEG/MEG activity in lower frequency bands associated with AD. Signorino et al. [4] found an increase in the EEG powers of delta and theta bands in $\mathrm{AD}$ patients compared with control subjects. Other study showed increased slower and reduced faster activity in AD patients' MEGs [5]. Nevertheless, the ability of human brain to perform sophisticated cognitive tasks supports the hypothesis that the brain may not be completely stochastic [6]. Moreover, nonlinearity is present in the brain, even at cellular level [7]. Therefore, non-linear analysis of EEG and MEG data might be a complementary tool to help physicians in the $A D$ diagnosis.

The first non-linear methods used to study the brain recordings from $\mathrm{AD}$ patients were correlation dimension (D2) and the first Lyapunov exponent (L1). Jeong et al. [8] demonstrated that $\mathrm{AD}$ patients exhibit significantly lower $D 2$ and $L 1$ values than controls in many EEG channels. Other study showed a decreased complexity of the MEG background activity in $\mathrm{AD}$ patients in the low frequency bands, and an increased in the high bands [9]. Nevertheless, these classical measures for complexity estimation have some drawbacks. Reliable estimation of $L 1$ and $D 2$ requires a large number of data points and stationary and noise-free time series $[8,10]$. As these problems cannot be solved for physiological signals, other non-linear methods are necessary to study brain recordings. EEG/MEG studies found that $\mathrm{AD}$ patients had significantly lower Lempel-Ziv complexity values than elderly control subjects $[11,12]$. Using approximate entropy, Abásolo et al. [13] found that AD patients' EEGs were more regular than control subjects' recordings. Moreover, Stam et al. [14] examined the fluctuation of the EEG synchronization level of AD patients with detrended fluctuation analysis.

In this study, we have examined the MEG background activity in $20 \mathrm{AD}$ patients and 21 control subjects with two non-linear methods: sample entropy (SampEn) and 
multiscale entropy (MSE). Our purpose was to test the hypothesis that entropy analyses of the magnetic brain activity would be different in both groups, hence indicating an abnormal type of dynamics associated with AD.

\section{MATERIALS AND METHODS}

\section{A. Subjects and $M E G$ recording}

The signals were acquired with a 148-channel whole-head magnetometer (MAGNES 2500 WH, 4D Neuroimaging) placed in a magnetically shielded room. The subjects lay on a patient bed, in a relaxed state and with their eyes closed. For each subject, MEG registration was performed with a 678.17 Hz sampling frequency, using a hardware band-pass filter of 0.1-200 Hz. Afterward, these recordings were downsampled by a factor of $4(169.549 \mathrm{~Hz}, 50863$ samples). Artifact-free epochs of 10 seconds (1696 data points) were selected. Finally, these epochs were filtered between 0.5 and $40 \mathrm{~Hz}$ and copied to a computer as ASCII files for further non-linear analysis.

In the present study, MEG signals were recorded from 41 subjects. Cognitive status was screened in both groups with Folstein's Mini Mental State Examination (MMSE). MEGs were obtained from twenty patients ( 7 men and 13 women; age $=73.05 \pm 8.65$ years, mean \pm standard deviation, SD) fulfilling the criteria of probable AD. They were recruited from the Asociación de Enfermos de Alzheimer (AFAL). Diagnosis for all patients was made according to the criteria of the National Institute of Neurological and Communicative Disorders and Stroke-Alzheimer's Disease and Related Disorders Association (NINCDS-ADRDA). The MMSE score for these patients was $17.85 \pm 3.91$ (mean \pm SD). Patients were free of other significant medical, neurological and psychiatric diseases than $\mathrm{AD}$ and they were not taking drugs which could affect MEG activity.

The control group consisted of 21 elderly control subjects without past or present neurological disorders (9 men and 12 women; age $=70.29 \pm 7.07$ years, MMSE score $=29.10 \pm$ 1.00 points, mean \pm SD). All control subjects and all patients' caregivers signed an informed consent for the participation in this research work. The local Ethics Committee approved this study.

\section{B. Sample entropy (SampEn)}

SampEn is an embedding entropy that quantifies the regularity of a signal [15]. SampEn is the negative natural logarithm of the conditional probability that two sequences similar for $m$ points remain similar at the next point, where self-matches are not included in calculating the probability [15]. To compute SampEn, two input parameters must be specified: a run length $m$ and a tolerance window $r$. In our study, we have chosen $m=1$ and $r=0.25$ times the standard deviation of the original time series, due to these values have been previously used in other AD study [16]. This measure has already been used to study some biological signals. Applied to neonatal heart rate recordings, SampEn values fall before clinical signs of sepsis [17]. Kim et al. [18] investigated the non-linear characteristics of heart rate variability for different recumbent positions using SampEn.

Given a one dimensional time series $X=x(1), x(2), \ldots x(N)$, we describe the algorithm to compute the SampEn [15]:

1) Form $N-m+1$ vectors $X_{m}(i)$ defined by: $X_{m}(i)=X_{m}(i+$ $k$ ) with $0 \leq k \leq m-1$.

2) The distance between two of this vectors is the maximum difference of their corresponding scalar components:

$$
d\left[X_{m}(i), X_{m}(j)\right]=\max (|x(i+k)-x(j+k)|)
$$

for $0 \leq k \leq m-1$.

3) Define $B_{i}{ }^{m}(r)$ as $1 /(N-m-1)$ times the number of vectors $X_{m}(j)$ within $r$ of $X_{m}(i)$, where $1 \leq j \leq N-m(j \neq$ $i)$. Then, set $B_{m}(r)$ as:

$$
B_{m}(r)=\frac{1}{N-m} \sum_{i=1}^{N-m} B_{i}^{m}(r)
$$

4) Similarly, calculate $A_{i}{ }^{m}(r)$ as $1 /(N-m-1)$ times the number of $j(1 \leq j \leq N-m ; j \neq i)$, such the distance between $X_{m+1}(j)$ and $X_{m+1}(i)$ is less than or equal to $r$. Set $A_{m}(r)$ as:

$$
A_{m}(r)=\frac{1}{N-m} \sum_{i=1}^{N-m} A_{i}^{m}(r)
$$

5) Finally, we define:

$$
\operatorname{SampEn}(m, r)=\lim _{N \rightarrow \infty}\left[-\ln \frac{A_{m}(r)}{B_{m}(r)}\right]
$$

which is estimated by the statistic

$$
\operatorname{SampEn}(m, r, N)=-\ln \frac{A_{m}(r)}{B_{m}(r)}
$$

\section{Multiscale entropy (MSE)}

$M S E$ is a non-linear technique to measure complexity over a range of scales [19]. It is based on successive computations of the SampEn estimated on coarse-grained time series. MSE has been used to analyze cardiac interbeat interval time series from healthy subjects, patients with severe congestive heart failure and subjects with atrial fibrillation [20]. Costa et al. [19] found that spontaneous output of the human locomotor system during usual walking is more complex that walking under slow, fast or metronomically-paced protocols. Coding and non-coding DNI sequences have also been analyzed with this complexity measure [21]. In addition, Escudero et al. [22] examined the EEG background activity of AD patients and control subjects using $M S E$.

The MSE algorithm is as follows [20]. For a time series $X$ $=x(1), x(2), \ldots x(N)$, consecutive coarse-grained time series $Y^{\tau}=y^{\tau}(1), y^{\tau}(2), \ldots, y^{\tau}(N / \tau)$ should be constructed, according to:

$$
y^{\tau}(j)=\frac{1}{\tau} \sum_{i=(j-1) \tau+1}^{j \tau} x(i) \quad 1 \leq j \leq N / \tau
$$

Afterwards, SampEn for each coarse-grained sequence is 
calculated.

\section{Statistical analysis}

Firstly, a statistical analysis was carried out separately for each channel. Student's $t$-tests with the Bonferroni's correction were used to determine if there were any differences between SampEn and MSE values in both groups.

Secondly, mean values, calculated averaging the results from the 148 channels for each subject, were analyzed with receiver operating characteristic (ROC) curves. This statistical method summarizes the performance of a twoclass classifier across the range of possible thresholds. It is a graphical representation of the trade-offs between sensitivity and specificity. Sensitivity is the true positive rate while specificity is equal to the true negative rate:

$$
\begin{aligned}
& \text { Sensitivity }=\frac{T P}{T P+F N} \\
& \text { Specificity }=\frac{T N}{T N+F P}
\end{aligned}
$$

where false negatives $(F N)$ are the $\mathrm{AD}$ patients classified as control subjects, and false positives $(F P)$ are the controls classified as patients. True positives $(T P)$ and true negatives (TN) are the patients and control subjects correctly recognized, respectively. The area under the ROC curve (AROC) is a single number summary of performance. For a perfect test the area is 1 while an AROC of 0.5 represents a worthless test.

\section{RESUlts}

SampEn algorithm were applied for the 148 MEG channels with $m=1$ and $r=0.25$ times the standard deviation of the original time series. Our results showed that SampEn values were higher in the control group than in the AD patients group for all channels. Moreover, the differences were statistically significant in 16 channels $(p<$ 0.01 , Student's $t$-test with Bonferroni's correction).

We next apply the MSE method to the MEG recordings with the same values of $m$ and $r$. MSE profiles were obtained representing the SampEn values of each coarse-

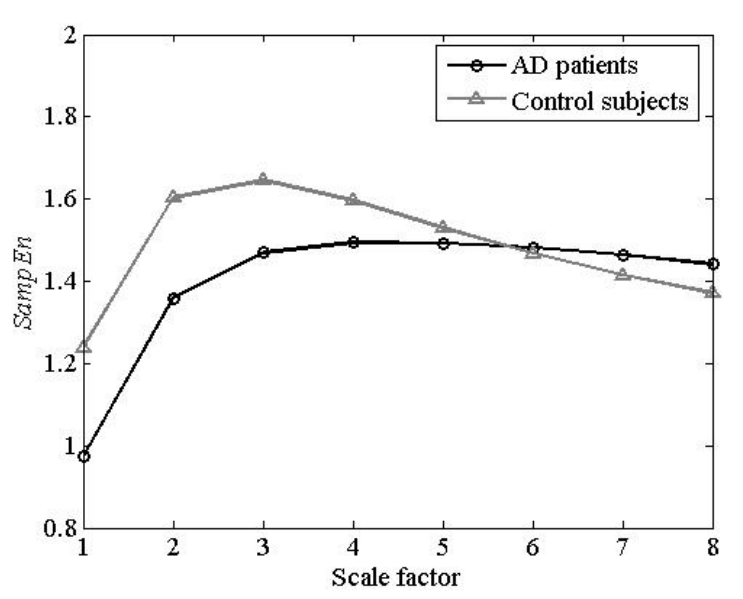

Fig. 1. MSE analysis of the $20 \mathrm{AD}$ patients (black curve) and the 21 control subjects (grey curve) with $m=1$ and $r=0.25$ times the standard deviation of the original data sequence. The curves were plotted averaging the results of the 148 MEG channels.

grained time series versus the scale factor. These profiles increased on the smaller time scales and then progressively decreased. Fig. 1 shows this performance for the mean values of each group, averaging the $M S E$ results of all channels. We estimated the slopes of the profiles for small (1 $\leq$ scale factor $\leq 4)$ and large time scales $(4 \leq$ scale factor $\leq$ 8 ) by means of the least-squares method. For each MEG channel, we compared the slopes of the AD patients group and the control subjects group. In 46 channels, significant differences between both groups were found $(p<0.01$, Student's $t$-test, Bonferroni's correction) when the slopes for small time scales were analyzed. Moreover, the comparison of the slopes for large time scales revealed significant differences in 99 MEG channels $(p<0.01$, Student's $t$-test, Bonferroni's correction).

Finally, ROC curves were used to assess the ability of our methods to discriminate AD patients from control subjects (Fig. 2). Mean values, obtained averaging the results of all channels, were used in this statistical analysis. With SampEn results, sensitivity of $80 \%$ and a specificity of $76.2 \%$ where achieved at the optimum threshold (1.11). Using the average slopes of the MSE profiles for small time scales, we obtained

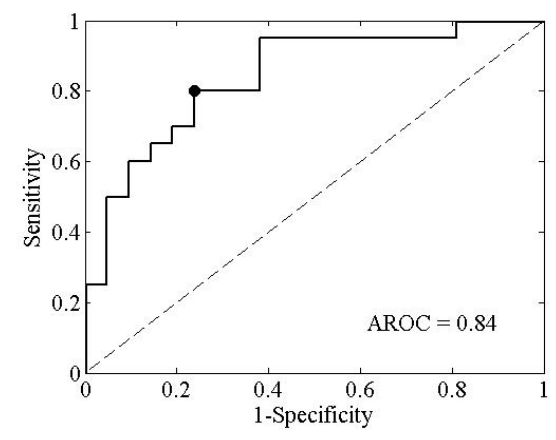

(a)

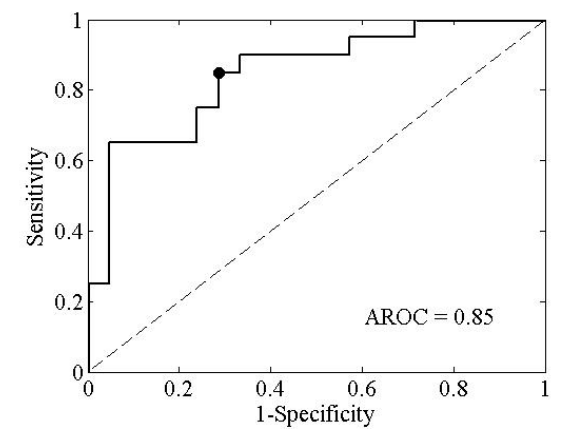

(b)

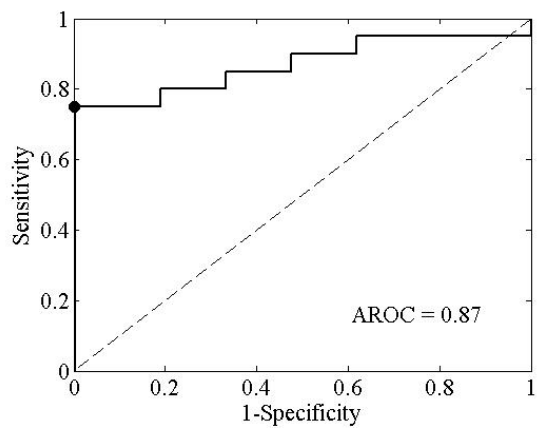

(c)

Fig. 2. ROC curves showing the discrimination between AD patients and control subjects with the mean SampEn values (a), and with the average slope values of the MSE profiles for small (b) and large time scales (c). A solid circle indicates the optimum cut-off point for each ROC curve. 
$85 \%$ sensitivity and $71.4 \%$ specificity at an optimum threshold of 0.13 . The best results were achieved when the slopes for large scales were analyzed. An accuracy of $87.8 \%$ ( $75 \%$ sensitivity, $100 \%$ specificity) was reached at a cut-off point of -0.02 .

\section{DISCUSSION AND CONCLUSIONS}

We have studied the MEG background activity of $20 \mathrm{AD}$ patients and 21 elderly control subjects with SampEn and $M S E$. Our purpose was to check the hypothesis that the brain activity recorded in MEG signals was different in $\mathrm{AD}$ patients than in control subjects.

Our results revealed that AD patients have lower SampEn values at all channels, indicating an increase of the MEG regularity associated with the disease. Our results are in agreement with previous research works that have applied non-linear methods to study the regularity of the brain activity in $\mathrm{AD}$ patients $[13,16]$. Approximate entropy values were significantly lower in the EEG of AD patients at $\mathrm{P} 3$ and P4 [13]. With SampEn, statistically significant differences between AD patients and controls were found at parietal and occipital EEG electrodes [16].

The MSE analysis showed that $\mathrm{AD}$ patients have lower SampEn values on the small and medium time scales. If SampEn values are higher for one signal than for another at most scale factors, we can assert that the former is more complex than the latter [21]. Thus, our study suggests that brains affected by $\mathrm{AD}$ show a less complex physiological behaviour. These results agree with other studies that showed a decreased complexity in the brain recordings of $\mathrm{AD}$ patients. For instance, Abásolo et al. [11] found significant differences in some EEG channels with LempelZiv complexity. This non-linear complexity measure has also been applied to MEG data [12]. Traditional non-linear methods, $D 2$ and $L 1$, also have been used to estimate the complexity of EEG/MEG recordings $[8,9]$.

Some limitations of our study merit consideration. The sample size is small. Thus, a larger database is needed to confirm our results. Moreover, the detected decreased in irregularity and complexity may not be specific to $\mathrm{AD}$ and it appears in other pathological states. Finally, our results do not show if SampEn and MSE can detect a gradation of the disease process.

In conclusion, non-linear analysis of the MEG background activity with SampEn and MSE revealed an increased regularity and a decreased complexity of the $\mathrm{AD}$ patients' recordings. Our results suggest that neuronal dysfunction in $\mathrm{AD}$ is associated with differences in the dynamical processes underlying the MEG recording.

\section{REFERENCES}

[1] M. Hämäläinen, R. Hari, R. J. Ilmoniemi, J. Knuutila, O. V. Lounasmaa, "Magnetoencephalography - theory, instrumentation, and applications to noninvasive studies of the working human brain," Rev. Mod. Phys., vol. 65, pp. 413-497, 1993.

[2] T. D. Bird, "Alzheimer's disease and other primary dementias," in Harrison's principles of internal medicine, E. Braunwald, A. S. Fauci,
D. L. Kasper, S. L. Hauser, D. L. Longo, and J. L. Jameson, Eds. New York: The McGraw-Hill Companies Inc, 2001, pp. 2391-2399.

[3] P. R. Hof and J. H. Morrison, "The cellular basis of cortical disconnection in Alzheimer disease and related dementing conditions," in Alzheimer disease, R. D. Terry, R. Katzman, K. L. Bick, and S. S. Sisodia, Eds. New York: Lippincott Willimas and Wilkins, 1999, pp. 207-232.

[4] M. Signorino, E. Pucci, N. Belardinelli, G. Nolle, and F. Angeleri, "EEG spectral analysis in vascular and Alzheimer dementia," Electroencephalogr. Clin. Neurophysiol., vol. 94, pp. 313-325, 1995.

[5] D. Osipova, J. Ahveninen, O. Jensen, A. Ylikoski, and E. Pekkonen, "Altered generation of spontaneous oscillations in Alzheimer's disease," Neuroimage, vol. 27, pp. 835-841, 2005.

[6] X. S. Zhang, R. J. Roy, and E. W. Jensen, "EEG complexity as a measure of depth of anesthesia for patients," IEEE Trans. Biomed. Eng., vol. 48, pp. 1424-1433, 2001.

[7] R. G. Andrzejak, K. Lehnertz, F. Mormann, C. Rieke, P. David, and C.E. Elger, "Indications of nonlinear deterministic and finitedimensional structures in time series of brain electrical activity: Dependence on recording region and brain state," Phys. Rev. E, vol. 64, p. 061907, 2001.

[8] J. Jeong, S. J. Kim, and S. H. Han, "Non-linear dynamical analysis of the EEG in Alzheimer's disease with optimal embedding dimension," Electroencephalogr. Clin. Neurophysiol., vol. 106, pp. 220-228, 1998.

[9] A. M. van Cappellen van Walsum, Y. A. L. Pijnenburg, H. W. Berendse, B. W. van Dijk, D. L. Knol, Ph. Scheltens, and C. J Stam, "A neural complexity measure applied to MEG data in Alzheimer's disease," Clin. Neurophysiol., vol. 114, pp. 1034-1040, 2003.

[10] J. P. Eckmann and D. Ruelle, "Fundamental limitations for estimating dimensions and Lyapunov exponents in dynamical systems," Physica $D$, vol. 56, pp. 185-187, 1992.

[11] D. Abásolo, R. Hornero, C. Gómez, M. García, and M. López, "Analysis of EEG background activity in Alzheimer's disease patients with Lempel-Ziv complexity and Central Tendency Measure," Med. Eng. Phys., vol. 28, pp. 315-322, 2006.

[12] C. Gómez, R. Hornero, D. Abásolo, A. Fernández, and M. López, "Complexity analysis of the magnetoencephalogram background activity in Alzheimer's disease patients," Med. Eng. Phys., vol. 28, pp. $851-859,2006$.

[13] D. Abásolo, R. Hornero, P. Espino, J. Poza, C.I. Sánchez, and R. de la Rosa, "Analysis of regularity in the EEG background activity of Alzheimer's disease patients with aproximate entropy," Clin. Neurophysiol., vol. 116, pp. 1826-1834, 2005.

[14] C. J. Stam, T. Montez, B. F. Jones, S. A. R. B. Rombouts, Y. van der Made, Y. A. L. Pijnenburg, and Ph. Scheltens, "Disturbed fluctuations of resting state EEG synchronization in Alzheimer's disease," Clin. Neurophysiol., vol. 116, pp. 708-715, 2005.

[15] J. S. Richman and J R. Moorman, "Physiological time-series analysis using approximate entropy and sample entropy," Am. J. Physiol. Heart Circ. Physiol., vol. 278, pp. H2039-H2049, 2000.

[16] D. Abásolo, R. Hornero, P. Espino, D. Álvarez, and J. Poza, "Entropy analysis of the EEG background activity in Alzheimer's disease patients," Physiol. Meas., vol. 27, pp. 241-253, 2006.

[17] D. E. Lake, J. S. Richman, M. P. Griffin, and J. R. Moorman, "Sample entropy analysis of neonatal heart rate variability," Am. J. Physiol. Regul. Integr. Comp. Physiol., vol. 282, pp. R789-R797, 2002.

[18] W.-S. Kim, Y.-Z. Yoon, J.-H. Bae, and K.-S. Soh, "Nonlinear characteristics of heart rate time series: influence of three recumbent positions in patients with mild or severe coronary artery disease," Physiol. Meas., vol. 26, pp. 517-529, 2005.

[19] M. Costa, C.-K. Peng, A. L. Goldberger, and J. M. Hausdorff, "Multiscale entropy analysis of human gait dynamics," Physica D, vol. 330, pp. 53-60, 2003.

[20] M. Costa, A. L. Goldberger, and C.-K. Peng, "Multiscale entropy analysis of complex physiologic time series," Phys. Rev. Lett., vol. 89, p. 068102, 2002.

[21] M. Costa, A. L. Goldberger, and C.-K. Peng, "Multiscale entropy analysis of biological signals," Phys. Rev. E, vol. 71, pp. 021906(18), 2005

[22] J. Escudero, D. Abásolo, R. Hornero, P. Espino, and M. López, "Analysis of electroencephalograms in Alzheimer's disease patients with multiscale entropy," Physiol. Meas., vol. 27, pp. 1091-1106, 2006. 